Géométrie algébrique/Algebraic geometry

\title{
Un théorème du point fixe de Lefschetz en géométrie d'Arakelov
}

\section{Kai KÖHLER}

Mathematisches Institut, Wegelerstr. 10, D-53115 Bonn, Allemagne, E-mail: koehler@rhein.iam.uni-bonn.de

\section{Damian ROESSLER}

Institut fuer Mathematik, Unter den Linden 6, 10099 Berlin, Allemagne, E-mail: roessler@mathematik.hu-berlin.de

Résumé- On considère des variétés arithmétiques munies d'une action du schéma en groupes des n-ième racines de l'unité et on défnit la $K_{0}$-théorie arithmétique équivariante pour ces variétés. On énonce ensuite un théorème de Riemann-Roch pour la transformation naturelle de la $K_{0}$-théorie arithmétique équivariante induite par la restriction au schéma des points fixes et on montre qu'il implique une version de la conjecture de Bismut d'un théorème de Riemann-Roch arithmétique équivariant.

\section{A Lefschetz fixed point theorem in Arakelov geometry}

\begin{abstract}
We consider arithmetic varieties endowed with an action of the group scheme of $n$-th roots of unity and we define equivariant arithmetic $K_{0}$-theory for these varieties. We then state a Riemann-Roch theorem for the natural transformation of equivariant arithmetic $K_{0}$-theory induced by the restriction to the fixed point scheme and we show that it implies a version of Bismut's conjecture of an equivariant arithmetic Riemann-Roch theorem.
\end{abstract}

\section{Préliminaires}

Soit $\mu_{n}:=\operatorname{Spec} \mathbf{Z}[T] /\left(1-T^{n}\right)$ le schéma en groupes des $n$-ièmes racines de l'unité. On fixe un générateur $g$ de $\mu_{n}(\mathbf{C})$. Un schéma $\mu_{n}$-équivariant admettant une immersion fermée équivariante dans un espace projectif muni d'une action de $\mu_{n}$ sera dit $\mu_{n}$-projectif (sur $\mathbf{Z}$ ). Soit $f: Y \rightarrow \mathbf{Z}$ un schéma régulier, $\mu_{n}$-projectif et plat sur $\mathbf{Z}$. On dénote par $Y_{\mu_{n}}$ le sous-schéma fermé des points fixes de $Y$; c'est un schéma régulier (voir [10, Prop. 3.1, p. 455]) que l'on supposera plat sur $\mathbf{Z}$ pour simplifier. On écrira $Y(\mathbf{C})$ pour la variété analytique des points complexes de $Y$. Cette variété porte par construction l'action holomorphe du groupe $\mu_{n}(\mathbf{C})$ des $n$-ièmes racines de l'unité. La conjugaison complexe induit un automorphisme antiholomorphe $F_{\infty}$ de $Y(\mathbf{C})$. On définit $A^{p, p}\left(Y_{\mu_{n}}\right)$ comme l'ensemble des formes différentielles complexes $\omega$ de type $(p, p)$ sur $Y_{\mu_{n}}(\mathbf{C})$ satisfaisant l'équation $F_{\infty}^{*} \omega=(-1)^{p} \omega$. On pose alors $\widetilde{A}\left(Y_{\mu_{n}}\right):=\oplus_{p} \geq 0\left(A^{p, p}\left(Y_{\mu_{n}}\right) /(\operatorname{Im} \partial+\operatorname{Im} \bar{\partial})\right)$.

On appelle fibré hermitien équivariant $\bar{E}:=(E, h, a)$ la donnée d'un fibré vectoriel algébrique sur $Y$ muni d'une action $a$ de $\mu_{n}$ relevant l'action de $\mu_{n}$ sur $Y$ (voir [11, Par. 1]) et d'une métrique hermitienne $h$ sur le fibré holomorphe $E_{\mathbf{C}}$ associé à $E$ sur $Y(\mathbf{C})$, invariante par $F_{\infty}$ et $a$. Si l'action de $\mu_{n}$ sur $Y$ est triviale, on appellera simplement fibré hermitien, un fibré hermitien équivariant muni également d'une action triviale.

Fixons un fibré hermitien équivariant $\bar{E}$. Les propriétés des schémas en groupes diagonalisables (voir [9, Par. 3.4, p. 47]) montrent que la donnée de l'action de $\mu_{n}$ sur la restriction $\left.E\right|_{Y_{\mu_{n}}}$ de $E$ à $Y_{\mu_{n}}$ est équivalente à la donnée d'une $\mathbf{Z} /(n)$-graduation sur $\left.E\right|_{Y_{\mu_{n}}}$. Les termes de cette graduation sont orthogonaux entre eux. On obtient ainsi une décomposition canonique en somme directe orthogonale de fibrés hermitiens équivariants $\bar{E}_{m}:\left.\bar{E}\right|_{Y_{\mu_{n}}} \simeq \bigoplus_{m \in \mathbf{Z} /(n)} \bar{E}_{m}$. Par ailleurs, écrivons $\Omega^{E} \in C^{\infty}\left(Y(\mathbf{C}), \operatorname{End}\left(E_{\mathbf{C}}\right) \otimes A^{1,1}(Y(\mathbf{C}))\right)$ pour la forme de courbure associée à l'unique 
connexion de $E_{\mathbf{C}}$ qui est de type $(1,0)$ et est compatible avec la structure hermitienne. On écrira alors $\operatorname{ch}_{g}(\bar{E})$ pour la forme différentielle complexe $\operatorname{Tr}\left(g \cdot \exp \left(\frac{i}{2 \pi} \Omega^{E}\right)\right)$ sur $Y_{\mu_{n}}(\mathbf{C})$, où $g$ dénote l'automorphisme de $\left.E_{\mathbf{C}}\right|_{Y_{\mu_{n}}(\mathbf{C})}$ défini par l'élément $g \in \mu_{n}(\mathbf{C})$ fixé plus haut. Dénotons par $\bar{E}_{m \neq 0}$ le fibré hermitien équivariant $\bigoplus_{m \in \mathbf{Z} /(n), m \neq 0} \bar{E}_{m}$. On écrira alors $\operatorname{Todd}_{g}(\bar{E})$ pour la forme différentielle $\operatorname{Todd}\left(\bar{E}_{0}\right) \cdot\left(\sum_{i=0}^{r g\left(E_{m \neq 0}\right)}(-1)^{i} \operatorname{ch}_{g}\left(\Lambda^{i}\left(\bar{E}_{m \neq 0}^{\vee}\right)\right)\right)^{-1}$. Ici Todd $\left(\bar{E}_{0}\right)$ est la forme de Todd associée par les formules de Chern-Weil à l'unique connexion de $E_{0 \mathbf{C}}$ qui est de type $(1,0)$ et est compatible avec la structure hermitienne; $\Lambda^{i}\left(\bar{E}_{m \neq 0}^{\vee}\right)$ est la i-tième puissance extérieure du dual de $\bar{E}_{m \neq 0}$, munie de sa structure équivariante et hermitienne naturelle. Si $\mathcal{E}: 0 \rightarrow E^{\prime} \rightarrow E \rightarrow E^{\prime \prime} \rightarrow 0$ est une suite exacte de fibrés vectoriels équivariants sur $Y$ et $\overline{\mathcal{E}}$ est la donnée de $\mathcal{E}$ et de métriques hermitiennes invariantes par $g$ et $F_{\infty}$ sur $E_{\mathbf{C}}^{\prime}, E_{\mathbf{C}}$ et $E_{\mathbf{C}}^{\prime \prime}$, on dispose d'une classe de Bott-Chern secondaire équivariante $\widetilde{c h}(\overline{\mathcal{E}}) \in \tilde{A}\left(Y_{\mu_{n}}\right)$ (cf. [1, VI]).

\section{Le théorème}

Définition. Le groupe de Grothendieck arithmétique équivariant $\widehat{K}_{0}^{\mu_{n}}(Y)$ de $Y$ est le groupe abélien libre engendré par les éléments de $\widetilde{A}\left(Y_{\mu_{n}}\right)$ et par les classes d'isomorphismes isométriques équivariants de fibrés hermitiens équivariants, soumis aux relations

(a) Pour toute suite exacte $\overline{\mathcal{E}}$ comme plus haut, on a $\widetilde{c h}(\overline{\mathcal{E}})=\bar{E}^{\prime}-\bar{E}+\bar{E}^{\prime \prime}$.

(b) Si $\eta \in \widetilde{A}\left(Y_{\mu_{n}}\right)$ est la somme dans $\widetilde{A}\left(Y_{\mu_{n}}\right)$ de $\eta^{\prime}$ et $\eta^{\prime \prime}$, alors $\eta=\eta^{\prime}+\eta^{\prime \prime}$ dans $\widehat{K}_{0}^{\mu_{n}}(Y)$.

Choisissons maintenant une métrique de Kähler sur $Y(\mathbf{C})$, invariante par $\mu_{n}(\mathbf{C})$ et $F_{\infty}$. Soit $\omega_{Y}$ sa forme de Kähler. On peut alors associer à tout fibré hermitien equivariant $\bar{E}$ sur $Y$ sa torsion analytique equivariante $T_{g}\left(\omega_{Y}, \bar{E}\right) \in \mathbf{C}$ (pour la déf., voir [8, Par. 1]). Soit $(E, h, a)$ un fibré hermitien équivariant sur $Y$ tel que $E$ est acyclique et soit $\eta \in \widetilde{A}\left(Y_{\mu_{n}}\right)$. On dénote par $f_{*} E$ le $\mathbf{Z}$ module de type fini qui est l'image directe de $E$, par $f_{*} a$ la structure équivariante de $f_{*} E$ héritée de $E$ par fonctorialité et enfin on dénote par $f_{*} h$ la métrique de $\left(f_{*}(E)\right)_{\mathbf{C}}$ héritée de $E$ par integration le long des fibres (pour la déf., voir [12, 9.2, p. 278]). On démontre qu'il existe un unique morphisme de groupes $f_{*}: \widehat{K}_{0}^{\mu_{n}}(Y) \rightarrow \widehat{K}_{0}^{\mu_{n}}(\mathbf{Z})$, tel que $f_{*}(\bar{E}+\eta)=\left(f_{*} E, f_{*} a, f_{*} h\right)-T_{g}\left(\omega_{Y},(E, a, h)\right)+$ $\int_{Y(\mathbf{C})} \operatorname{Todd}_{g}\left(\overline{T Y_{\mathbf{C}}}\right) \eta$ dans $\widehat{K}_{0}^{\mu_{n}}(\mathbf{Z})$, pour tout fibré hermitien équivariant $\bar{E}$ comme plus haut.

Par ailleurs, soit $\theta \in \mathbf{R}$. Pour $s \in \mathbf{C}$ avec $s>0$, on définit $\zeta(\theta, s):=\sum_{n \geq 1} \frac{\cos (n \theta)}{n^{s}}$ et $\eta(\theta, s):=$ $\sum_{n \geq 1} \frac{\sin (n \theta)}{n^{s}}$. Soit $R(\theta, t)$ la série formelle de puissances

$$
\sum_{n \geq 1, n \text { impair }}\left(2 \zeta^{\prime}(\theta,-n)+\sum_{j=1}^{n} \frac{\zeta(\theta,-n)}{j}\right) \frac{t^{n}}{n !}+i . \sum_{n \geq 0, n \text { pair }}\left(2 \eta^{\prime}(\theta,-n)+\sum_{j=1}^{n} \frac{\eta(\theta,-n)}{j}\right) \frac{t^{n}}{n !}
$$

(voir [2]). Nous aurons besoin de $R(\theta,(\cdot))$ qui est l'unique classe caractéristique additive définie sur les fibrés holomorphes telle que $R(\theta, L)=R\left(\theta, c^{1}(L)\right)$ pour tout fibré en droites $L$.

Soit $V$ un fibré vectoriel équivariant sur $Y$. Sur $Y_{\mu_{n}}(\mathbf{C})$, il existe une decomposition canonique en somme directe $\left.V_{\mathbf{C}}\right|_{Y_{\mu_{n}}(\mathbf{C})} \simeq \oplus_{k=1}^{r g(V)} V_{\theta_{k}}$ où $\theta_{1}, \ldots \theta_{r g(F)} \in\left[0,2 \pi\left[\right.\right.$ et $V_{\theta_{k}}$ est le plus grand sous-fibré de $\left.V_{\mathbf{C}}\right|_{Y_{\mu_{n}}(\mathbf{C})}$ sur lequel l'action relevant $g$ agit comme multiplication par $e^{i \theta_{k}}$. On définit $R_{g}(V):=$ $\sum_{k=1}^{r g(V)} R\left(\theta_{k}, V_{\theta_{k}}\right)$. Enfin, pour formuler le théorème du point fixe, on définit l'homomorphisme $\rho$ : $\widehat{K}_{0}^{\mu_{n}}(Y) \rightarrow \widehat{K}_{0}^{\mu_{n}}\left(Y_{\mu_{n}}\right)$; c'est l'unique homomorphisme qui associe à un fibré hermitien équivariant 
sa restriction à $Y_{\mu_{n}}$ et à un élément de $\widetilde{A}\left(Y_{\mu_{n}}\right)$ ce même élément. Soit encore $\bar{E}$ un fibré hermitien équivariant sur $Y$. On écrit $\lambda_{-1}(\bar{E})$ pour l'élément $\sum_{i=0}^{r g(E)}(-1)^{i} \Lambda^{i}(\bar{E})$ de $\widehat{K}_{0}^{\mu_{n}}(Y)$. Soit $R\left(\mu_{n}\right)$ le groupe de Grothendieck des $\mathbf{Z}$-modules projectifs et de type fini sur $\mathbf{Z}$ qui sont munis d'une structure de $\mu_{n}$-comodule. Il existe un isomorphisme canonique $R\left(\mu_{n}\right) \simeq \mathbf{Z}[T] /\left(1-T^{n}\right)$. On considère le corps $\mathbf{C}$ comme une $R\left(\mu_{n}\right)$-algèbre via l'unique morphisme d'anneaux $R\left(\mu_{n}\right) \rightarrow \mathbf{C}$ qui envoie $T$ sur $g$. Soit maintenant le fibré hermitien $\bar{F}$ tel que $F_{m}=0$ si $m \neq 1$ et tel que $\bar{F}_{1}$ est le fibré trivial $\mathcal{O}_{Y_{\mu_{n}}}$ muni de sa structure métrique triviale. On considère alors $\widehat{K}_{0}^{\mu_{n}}\left(Y_{\mu_{n}}\right)$ comme une $R\left(\mu_{n}\right)$ - algèbre via l'unique morphisme d'anneaux qui envoie $T$ sur $\bar{F}$. On fixe maintenant un élément $x \in \widehat{K}_{0}^{\mu_{n}}(Y)$. On dénote par $\bar{N}_{Y / Y_{\mu_{n}}}$ le fibré normal de $Y_{\mu_{n}}$ dans $Y$, muni de sa structure équivariante et métrique naturelle et on dénote par $f^{\mu_{n}}$ le morphisme $Y_{\mu_{n}} \rightarrow \mathbf{Z}$.

Théorème I.

(a) L'élément $\left(\lambda_{-1}\left(\bar{N}_{Y / Y_{\mu_{n}}}^{\vee}\right)\right)$ de l'anneau $\widehat{K}_{0}^{\mu_{n}}\left(Y_{\mu_{n}}\right) \otimes_{R\left(\mu_{n}\right)} \mathbf{C}$ possède un inverse multiplicatif.

(b) Le diagrame

$$
\begin{array}{ccc}
\widehat{K}_{0}^{\mu_{n}}(Y) & \left(\lambda_{-1}\left(\bar{N}_{Y / Y_{\mu_{n}}}^{\vee}\right)\right)^{-1} \cdot\left(1-R_{g}\left(N_{Y / Y_{\mu_{n}}}\right)\right) \cdot \rho(.) & \widehat{K}_{0}^{\mu_{n}}\left(Y_{\mu_{n}}\right) \otimes_{R\left(\mu_{n}\right)} \mathbf{C} \\
\downarrow f_{*} & \stackrel{I d \otimes 1}{\longrightarrow} & \downarrow f_{*}^{\mu_{n}} \\
\widehat{K}_{0}^{\mu_{n}}(\mathbf{Z}) & \stackrel{\widehat{K}_{0}^{\mu_{n}}(\mathbf{Z}) \otimes_{R\left(\mu_{n}\right)} \mathbf{C}}{\longrightarrow} & { }^{\longrightarrow}
\end{array}
$$

commute.

\section{Une application à la géométrie diophantienne}

Soit $\bar{V}$ un $\mathbf{Z}$-module hermitien. Le $\mathbf{Z}$-module $V$ forme un réseau dans le $\mathbf{R}$-espace vectoriel $V_{\mathbf{C}}^{+}$ qui est le sous-ensemble de $V \otimes_{\mathbf{z}} \mathbf{C}$ invariant par la conjugaison $F_{\infty}$. On appelle covolume de $\bar{V}$ et on écrit $\operatorname{covol}(\bar{V})$ pour le volume du quotient $V_{\mathbf{C}}^{+} / V$, calculé avec la forme volume héritée de la métrique de $V_{\mathbf{C}}^{+}$.

Soit $\bar{E}:=(E, a, h)$ un fibré hermitien équivariant sur $Y$. On suppose $E$ acyclique. L'élément $\left(f_{*} E, f_{*} a, f_{*} h\right)$ est alors représenté dans $\widehat{K}_{0}^{\mu_{n}}(\mathbf{Z})$ par le $\mu_{n}$-comodule hermitien $\overline{\Gamma(E)}$ des sections globales de $E$. On se propose de calculer en terme de classes caractéristiques arithmétiques (pour la définition de ce terme, voir [7]) le covolume de $\overline{\Gamma(E)}_{m}$, pour chaque $m \in \mathbf{Z} /(n)$.

Lemme.Il existe un unique morphisme de $R\left(\mu_{n}\right)$-modules mlcovol : $\widehat{K}_{0}^{\mu_{n}}(\mathbf{Z}) \rightarrow \mathbf{C}$, qui associe $\grave{a}$ un $\mu_{n}$-comodule hermitien $\bar{V}$ le nombre complexe $\sum_{m \in \mathbf{Z} /(n)}-\log \left(\operatorname{covol}\left(\bar{V}_{m}\right)\right) \cdot g^{m}$ et à $\eta \in \widetilde{A}(\mathbf{Z})$ le nombre complexe correspondant.

Soit $h: X \rightarrow \mathbf{Z}$ un schéma régulier, quasi-projectif et plat sur $\mathbf{Z}$. Par le symbole $\widehat{\mathrm{CH}}(X)$, on dénote l'anneau de Chow arithmétique de $X$ (voir [6]). A chaque fibré hermitien $\bar{V}$ sur $X$, on peut associer son caractère de Chern arithmétique $\widehat{\operatorname{ch}}(\bar{V}) \in \widehat{\mathrm{CH}}(X) \otimes_{\mathbf{z}} \mathbf{Q}$ (voir [7]). Pour chaque métrique de Kähler sur $X(\mathbf{C})$, il existe un élément $\widehat{\operatorname{Tod}} \mathrm{d}(\overline{T h}) \in \widehat{\mathrm{CH}}(X) \otimes_{\mathbf{Z}} \mathbf{Q}$, qui correspond à la classe de Todd arithmétique du fibré tangent lorsque $h$ est un morphisme lisse (voir [5, Prop. 1, p. 504]). Il existe aussi un morphisme de groupes $\widehat{\operatorname{deg}}: \widehat{\mathrm{CH}}(X) \rightarrow \mathbf{R}$ à valeurs réelles, appelé le degré arithmétique (voir $[4,2.5]$ ). Dans les prochaines définition et théorème, $E$ n'est pas nécessairement acyclique.

Définition. Le caractère de Chern arithmétique équivariant de $\bar{E}$ est l'élément $\widehat{c h}_{\mu_{n}}(\bar{E}):=\sum_{m \in \mathbf{Z} /(n)} \widehat{\operatorname{ch}}\left(\bar{E}_{m}\right) \otimes g^{m}$ de $\widehat{\mathrm{CH}}\left(Y_{\mu_{n}}\right) \otimes_{\mathbf{Z}} \mathbf{C}$. 
Le prochain théorème est une version de la conjecture de Bismut d'un théorème de Riemann-Roch arithmétique équivariant (voir [2]).

Théorème II. L'égalité de nombres complexes

$$
\begin{aligned}
\operatorname{mlcovol}\left(f_{*} E, f_{*} a, f_{*} h\right)-T_{g}\left(\omega_{Y}, \bar{E}\right)=\widehat{\operatorname{deg}}( & \left.\left(\sum_{i=0}^{r g\left(N_{Y / Y_{\mu_{n}}}^{\vee}\right)}(-1)^{i} \widehat{\operatorname{ch}}_{\mu_{n}}\left(\Lambda^{i}\left(\bar{N}_{Y / Y_{\mu_{n}}}^{\vee}\right)\right)\right)^{-1} \cdot \widehat{\operatorname{Tod}}\left(\overline{T f \mu^{\mu_{n}}}\right) \cdot \widehat{\operatorname{ch}_{\mu_{n}}}(\bar{E})\right)- \\
& \int_{Y_{\mu_{n}}(\mathbf{C})} \operatorname{Todd}_{g}\left(T Y_{\mathbf{C}}\right) \operatorname{ch}_{g}(E) R_{g}\left(T Y_{\mathbf{C}}\right)
\end{aligned}
$$

est vérifiée.

On suppose maintenant que $E$ est acyclique. On remarque qu'il existe une immersion de schémas en groupes $\mu_{n / p g c d(n, m)} \rightarrow \mu_{n}$ pour tout $m \in \mathbf{Z} /(n)$. Le schéma $Y$ ainsi que le fibré $E$ sont ainsi naturellement $\mu_{n / p g c d(n, m)}$-équivariants. Pour tout $m$, on choisit $g^{m}$ comme générateur de $\mu_{n / p g c d(n, m)}(\mathbf{C})$; on peut alors appliquer le théorème précèdent à chacun des $\mu_{n / p g c d(n, m)}$ successivement et on obtient un système de $n$ équations lineaires, qui est maximal et dont les inconnues sont les nombres $\log \left(\operatorname{covol}\left(\overline{\Gamma(E)_{l}}\right)\right)(l \in \mathbf{Z} /(n))$. On peut donc les déterminer explicitement.

\section{References}

[1] Bismut, J.-M.: Equivariant immersions and Quillen metrics. J. Differential Geom. 41, 53-157 (1995).

[2] Bismut, J.-M.: Equivariant short exact sequences of vector bundles and their analytic torsion forms. Comp. Math. 93, 291-354 (1994).

[3] Bismut, J.-M., Lebeau, G.: Complex immersions and Quillen metrics. Publications Math. IHES 74 (1990).

[4] Bost, J.-B.: Théorie de l'intersection et théorème de Riemann-Roch arithmétiques. Séminaire Bourbaki 43, n. 731 (1990).

[5] Gillet, H., Soulé, C.: An arithmetic Riemann-Roch theorem. Inventiones Math. 110, 473-543 (1992).

[6] Gillet, H., Soulé, C.: Arithmetic intersection theory. Publications Math. IHES 72 (1990).

[7] Gillet, H., Soulé, C.: Characteristic classes for algebraic vector bundles with hermitian metrics I, II. Annals of Math. 131, 163-203, 205-238 (1990).

[8] Köhler, K.: Equivariant analytic torsion on $\mathbf{P}^{n}(\mathbf{C})$. Math. Ann. 297, 553-565 (1993).

[9] Serre, J.-P.: Groupes de Grothendieck des schémas en groupes réductifs déployés. Publications Math. IHES 34, 37-52 (1968).

[10] Thomason, R.: Une formule de Lefschetz en $K$-théorie équivariante algèbrique. Duke Math. J. 68, 447-462 (1992).

[11] Thomason, R.: Algebraic K-theory of group scheme actions. Ann. of Math. Stud. 113, 539-563 (1987).

[12] Vergne, M., Berline, N., Getzler, E.: Heat kernels and Dirac operators: Springer 1992. 